\title{
Quantum Circuit Oscillator
}

This paper was downloaded from TechRxiv (https://www.techrxiv.org).

\section{LICENSE}

CC BY-NC-SA 4.0

SUBMISSION DATE / POSTED DATE

03-09-2021 / 11-09-2021

CITATION

Delgado, Alberto (2021): Quantum Circuit Oscillator. TechRxiv. Preprint.

https://www.techrxiv.org/articles/preprint/Quantum_Circuit_Oscillator/16566717

$\mathrm{DOI}$

10.36227/techrxiv.16566717.v1 


\title{
Quantum Circuit Oscillator
}

\author{
Alberto Delgado ${ }^{1}$, IEEE Senior Member
}

\begin{abstract}
There is an increasing interest to find applications of current quantum computers, small number of qubits and no error correction, known as NISQ computers. In this paper a parametrized quantum model is embedded in a chain of integrators to create an oscillator, the parameters are estimated using Taylor linearization and stability analysis from classic control systems theory.
\end{abstract}

\section{INTRODUCTION}

The idea of using quantum computers to simulate quantum systems was first proposed by Feynman in order to overcome the cost and limitations of using classical computers [1]. The mathematical foundations of quantum computing are well understood [2] and prototypes of quantum computers, with atoms or superconducting circuits representing quantum bits (qubits), are being developed [3]. Current quantum computers with around 50 qubits are called NISQ (Noisy Intermediate Scale Quantum) devices [1, 4], they are noisy because there is not error correction. There is a strong interest to learn about the capabilities of NISQ computers and to use them to develop future quantum computers, FTQC (Fault Tolerant Quantum Computing), that can solve computational tasks beyond classical devices [1].

On the other hand, machine learning is one approach to Artificial Intelligence (AI) where algorithms create models from data by tuning some parameters, the process is called learning from data or training the parametrized model. After training, models in operation are used to make predictions with many high impact applications in society [5].

There are two ways to incorporate machine learning into parametrized quantum circuits. The first approach is known as VQA (Variational Quantum Algorithm) and it has been used to solve linear systems of equations, integer factorization, and principal component analysis. The VQA requires an ansatz (parametrized quantum operation), training data, and a cost function to be optimized in order to solve the problem, the parameters are found with a classical optimizer [6]. The second approach has been called a kernel method or quantum model, this one requires mapping the data into quantum states and then performing a measurement as the model output. Training the quantum model means finding a measurement (prediction) to optimize a cost function that depends on data [7].

Following the idea to study NISQ computers, in particular applications in engineering, parametrized quantum circuits have been proposed to approximate nonlinear functions using data [8] and also synthesize the nonlinear current - voltage

\footnotetext{
${ }^{1}$ Alberto Delgado is with the Electrical and Electronics Engineering Department, National University of Colombia, Bogota, CO adelgadodieee.org
}

characteristic of a circuit device known as memristor [9]. These applications $[8,9]$ belong to the category of quantum models [7] where classical data is projected into a quantum state and a measurement is performed, parameters appear in the projection and also in the measurement, these can be set directly, calculated, or found with and optimizer and data.

The paper is organized as follows, section two presents the parametrized quantum model with the projection function and the measurement operator for the expectation value. Section three introduces the quantum model oscillator embedded in a chain of integrators and the corresponding simulation diagram. Section four applies Taylor linearization to the quantum model oscillator to find the parameters and understand the dynamics of the complete system. Section five includes three simulations, changing the quantum model parameters, to verify the theoretical analysis and to compare the dynamics of the nonlinear system with its Taylor linearization. Finally conclusions are formulated in section six.

\section{QUANTUM MODEL}

In the category of quantum models [7] lets consider a qubit in the quantum state,

$$
\mid \psi\left(x_{1}\right)>=\left[\begin{array}{l}
\cos \alpha\left(x_{1}\right) \\
\sin \alpha\left(x_{1}\right)
\end{array}\right]
$$

where the classical state $x_{1}$ is projected into the quantum state by using the angle function,

$$
\alpha\left(x_{1}\right)=\frac{\pi}{2} e^{-\frac{x_{1}^{2}}{s}}
$$

with a tunable parameter s.

The measurement is applied to the operator,

$$
G=\left[\begin{array}{cc}
g_{1} & 0 \\
0 & g_{2}
\end{array}\right]
$$

to obtain an expectation value,

$$
\begin{aligned}
& f\left(x_{1}\right)=<\psi\left(x_{1}\right)|G| \psi\left(x_{1}\right)> \\
& f\left(x_{1}\right)=g_{1} \cos \alpha\left(x_{1}\right)^{2}+g_{2} \sin \alpha\left(x_{1}\right)^{2}
\end{aligned}
$$

In words, the projection of a classical state $x_{1}$ into a quantum state, followed by a measurement and an operator results in a parametrized non linear function $f\left(x_{1}\right)$ with tunable parameters $\left\{g_{1}, g_{2}, s\right\}$.

\section{QUANTUM CIRCUIT OSCILLATOR}

The parametrized nonlinear function $f\left(x_{1}\right)$ is used in a classical dynamical system to create an oscillator, the frequency can be adjusted with parameters $\left\{g_{1}, g_{2}, s\right\}$. 


\section{A. Equations}

The classical dynamical system obeys the equations,

$$
\begin{aligned}
& \dot{x_{1}}=x_{2} \\
& \dot{x_{2}}=f\left(x_{1}\right)+b . u(t)
\end{aligned}
$$

in the analysis that follows only the autonomous system is considered, the input gain $b$ is set to zero.

\section{B. Simulation Diagram}

Equations (5) are represented with the simulation diagram shown in figure 1 ,

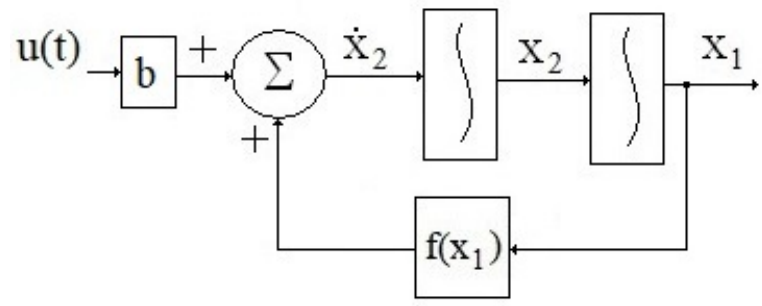

Fig. 1. Nonlinear oscillator using the quantum model $f\left(x_{1}\right)$.

System (5) is a chain of integrators with feedback that depends on $f\left(x_{1}\right)$, the nature of the feedback is determined by parameters $\left\{g_{1}, g_{2}, s\right\}$.

\section{TAYLOR LINEARIZATION}

As mentioned in the introduction, parameters in the quantum model can be set directly, calculated, or found with an optimizer and data. Here parameters are calculated using the Taylor linearization of system (5) around the equilibrium point [10].

\section{A. Equilibrium point}

System (5) has an equilibrium point when the time derivatives are zero, remember that $b=0$. Then,

$$
\begin{array}{r}
f\left(x_{1}\right)=0 \\
x_{2}=0
\end{array}
$$

solving for the equilibrium point $\left\{\bar{x}_{1}, \bar{x}_{2}\right\}$,

$$
\begin{aligned}
\tan \alpha\left(\bar{x}_{1}\right)^{2} & =-\frac{g_{1}}{g_{2}} \\
\bar{x}_{2} & =0
\end{aligned}
$$

From (7) a condition appears for $\left\{g_{1}, g_{2}\right\}$,

$$
-\frac{g_{1}}{g_{2}}>0
$$

\section{B. Linear system}

The linear version of system (5) can be written as,

$$
\frac{d}{d t}\left[\begin{array}{l}
z_{1} \\
z_{2}
\end{array}\right]=\left[\begin{array}{ll}
a_{11} & a_{12} \\
a_{21} & a_{22}
\end{array}\right]\left[\begin{array}{l}
z_{1} \\
z_{2}
\end{array}\right]
$$

where $a_{11}=a_{22}=0, a_{12}=1$.
To obtain the gain $a_{21}$ the derivative of $f\left(x_{1}\right)$ is calculated,

$$
\begin{aligned}
& a_{21}=\left.\frac{d f\left(x_{1}\right)}{d x_{1}}\right|_{\bar{x}_{1}} \\
& a_{21}=-\left[2\left(g_{1}-g_{2}\right) \sin \alpha\left(x_{1}\right) \cos \alpha\left(x_{1}\right) \frac{d \alpha\left(x_{1}\right)}{d x_{1}}\right]_{\bar{x}_{1}}
\end{aligned}
$$

where,

$$
\frac{d \alpha\left(x_{1}\right)}{d x_{1}}=-\frac{\pi x_{1}}{s} e^{-\frac{x_{1}^{2}}{s}}
$$

\section{Eigenvalues}

Using matrix A from linear system (9), the eigenvalues are calculated as $\operatorname{det}(\lambda I-A)=0$,

$$
\begin{aligned}
\left|\begin{array}{cc}
\lambda & -1 \\
-a_{21} & \lambda
\end{array}\right| & =0 \\
\lambda^{2} & =a_{21}
\end{aligned}
$$

From (12), to have complex conjugate roots the gain $a_{21}$ must be negative (10), this results in a second condition for $\left\{g_{1}, g_{2}\right\}$,

$$
g_{1}-g_{2}<0
$$

Finally, the linear oscillator frequency is,

$$
\omega=\sqrt{-a_{21}}
$$

In order to have an oscillator gains $\left\{g_{1}, g_{2}\right\}$ must satisfy conditions (8) and (13), the frequency $\omega$ can be controlled with the three parameters $\left\{g_{1}, g_{2}, s\right\}$.

\section{SIMULATIONS}

Following the analysis with the Taylor linearization of system (5), it is possible to set the parameters $\left\{g_{1}, g_{2}, s\right\}$ to create an oscillation with a given frequency.

Three simulations are considered as shown in Table 1, cases 1 and 2 illustrate the effects of parameter $s$ when parameters $\left\{g_{1}, g_{2}\right\}$ are fixed. Case 3 illustrates the effect when parameter $g_{2}$ is modified while keeping parameters $\left\{g_{1}, s\right\}$ fixed.

\begin{tabular}{||cccccc||}
\hline Case & $g_{1}$ & $g_{2}$ & $\bar{x}_{1}$ & $s$ & $a_{21}$ \\
\hline \hline 1 & -0.5 & 1.0 & 0.97 & 1.0 & -1.69 \\
\hline 2 & -0.5 & 1.0 & 0.68 & 0.5 & -2.38 \\
\hline 3 & -0.5 & 2.5 & 1.15 & 1.0 & -2.16 \\
\hline \multicolumn{7}{c}{ TABLE I } \\
PARAMETERS QUANTUM CIRCUIT OSCILLATOR.
\end{tabular}

The initial conditions for all simulations are,

$$
\begin{aligned}
& x_{1}(0)=\bar{x}_{1}+\Delta x, z_{1}(0)=\Delta x, \Delta x=0.2 \\
& x_{2}(0)=0, z_{2}(0)=0,
\end{aligned}
$$

the origin for the plot of the linearized system (9) is $\left\{\bar{x}_{1}, \bar{x}_{2}\right\}$.

Case 1 is used as reference to compare the increase in frequency: (i) when s decreases, case 2; (ii) when $\left|g_{1}-g_{2}\right|$ increases, case 3. 
A. Case 1: $g_{1}=-0.5, g_{2}=1.0, s=1.0$

Figure 2 shows the first state variable $\left\{x_{1}, z_{1}\right\}$ for systems (5) and (9), the initial condition is a perturbation $\Delta x$ from the equilibrium point. Figure 3 shows the second state variable $\left\{x_{2}, z_{2}\right\}$ for systems (5) and (9), initial conditions $x_{1}(0)=$ $z_{1}(0)=0$. These plots show Taylor linearization as a good approximation for the nonlinear system.

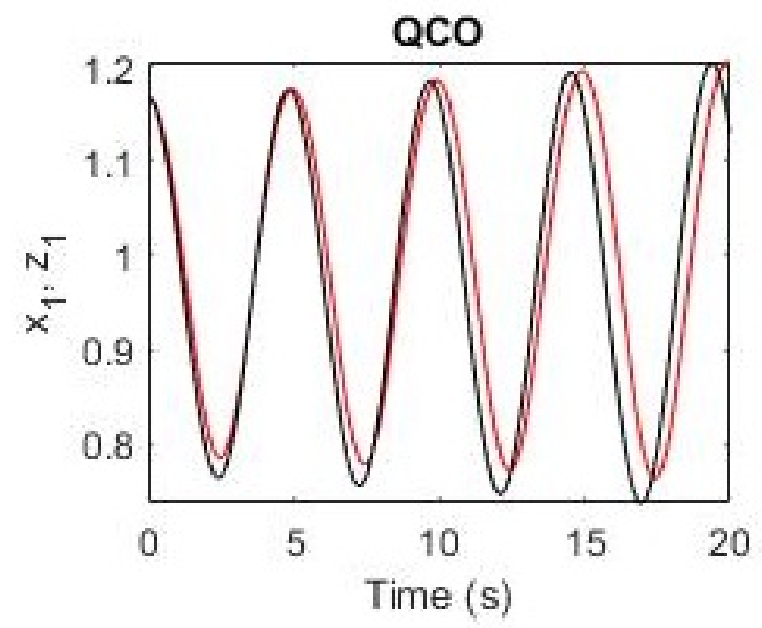

Fig. 2. $x_{1}$ (red) and $z_{1}$ from initial condition $x_{1}(0)=\bar{x}_{1}+\Delta x, z_{1}(0)=$ $\Delta x$. The linearized model (9) follows closely the nonlinear system (5).

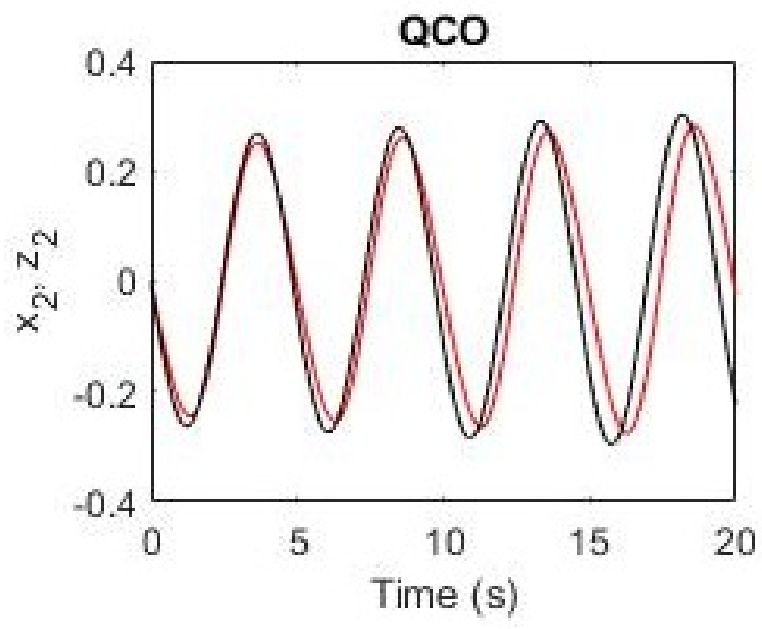

Fig. 3. $\quad x_{2}$ (red) and $z_{2}$ from initial condition $x_{2}(0)=0, z_{2}(0)=0$. The linearized model (9) follows closely the nonlinear system (5).

Figure 4 presents the phase plane for the nonlinear system (5) and Figure 5 shows the phase plane for the Taylor linearization (9), the state variables oscillate around the same region. It is important to mention again that the origin to plot system (9) is $\left\{\bar{x}_{1}, \bar{x}_{2}\right\}$. Figure 6 shows the angle $\alpha\left(x_{1}\right)$ changing in time, replacing (2) in (1) is a projection from the classical state $x_{1}$ to the quantum state $\left|\psi\left(x_{1}\right)\right\rangle$, probabilities for measuring the qubit oscillate.

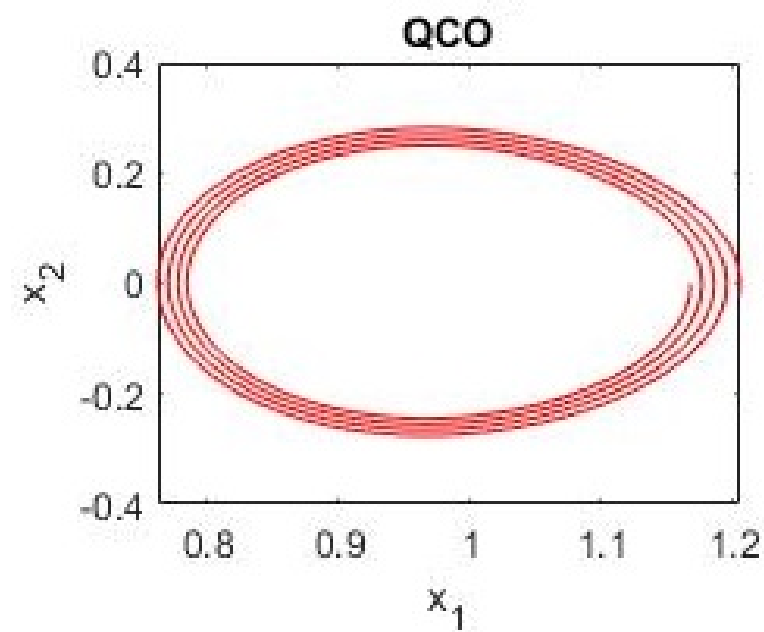

Fig. 4. Phase plot from initial condition $x_{1}(0)=\bar{x}_{1}+\Delta x, x_{2}(0)=0$. Notice that $x_{1}$ is positive but $x_{2}$ takes positive and negative values.

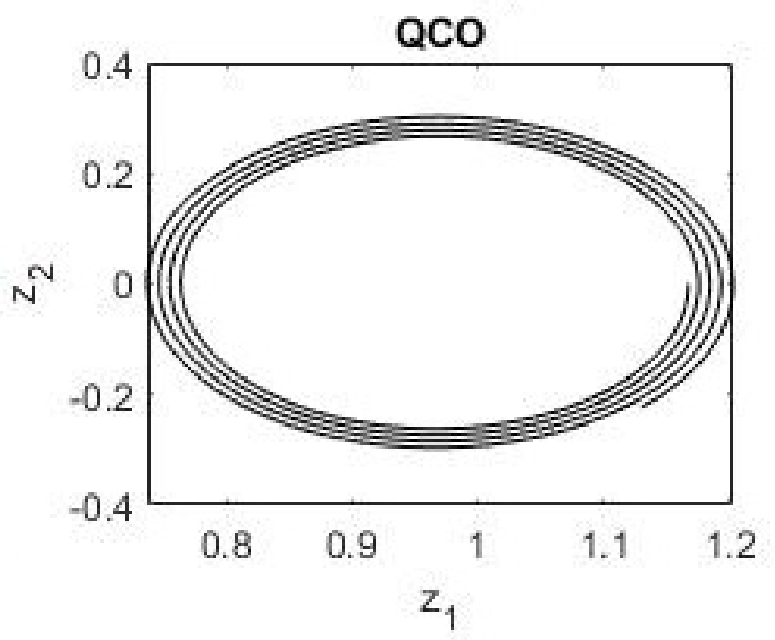

Fig. 5. Phase plot from initial condition $z_{1}(0)=\Delta x, z_{2}(0)=0$. Notice that $z_{1}$ is positive but $z_{2}$ takes positive and negative values.

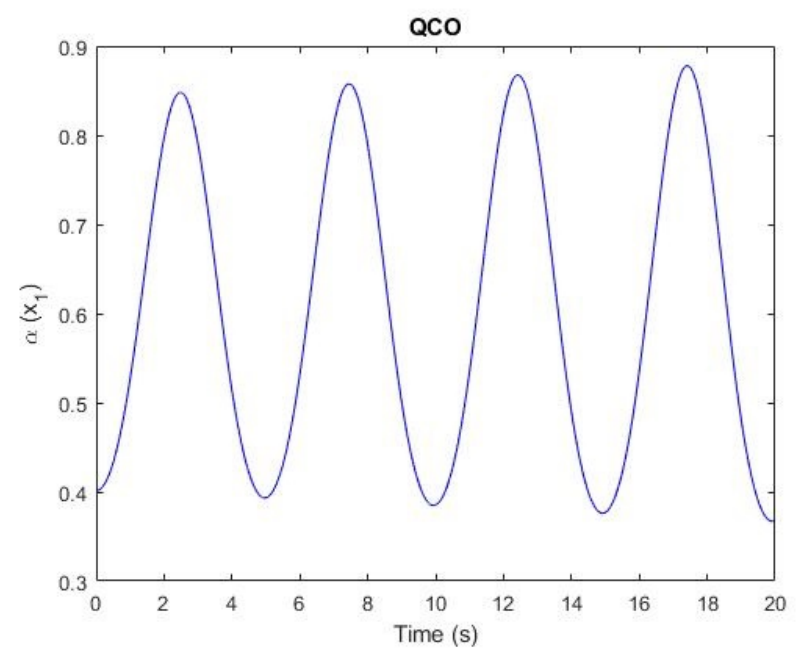

Fig. 6. $\alpha\left(x_{1}\right)$ as a function of time, note the periodicity. Probabilities for the qubit quantum states follow this trend. 


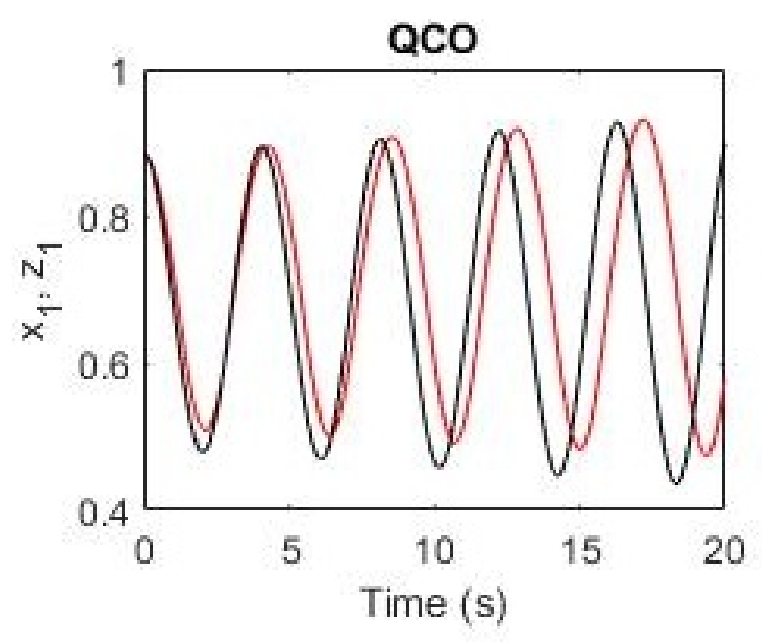

Fig. 7. Parameter $\mathrm{s}$ is reduced: $x_{1}$ (red) and $z_{1}$ from initial condition $x_{1}(0)=\bar{x}_{1}+\Delta x, z_{1}(0)=\Delta x$. There is an increase in frequency in relation with case 1 .

B. Case 2: $g_{1}=-0.5, g_{2}=1.0, s=0.5$

Parameter $\mathrm{s}$ is decreased, this causes an increase in frequency $\omega$.

Figure 7 shows the state variables $\left\{x_{1}, z_{1}\right\}$ for systems (5) and (9), the initial condition is a perturbation $\Delta x$ from $\left\{\bar{x}_{1}, \bar{x}_{2}\right\}$. Figure 8 shows the state variables $\left\{x_{2}, z_{2}\right\}$ for systems (5) and (9), initial conditions $x_{1}(0)=z_{1}(0)=0$. A comparison between case 1, figures 2 and 3, and case 2, figures 7 and 8 , shows an increase in frequency as expected (10) by the analysis of the linearized system.

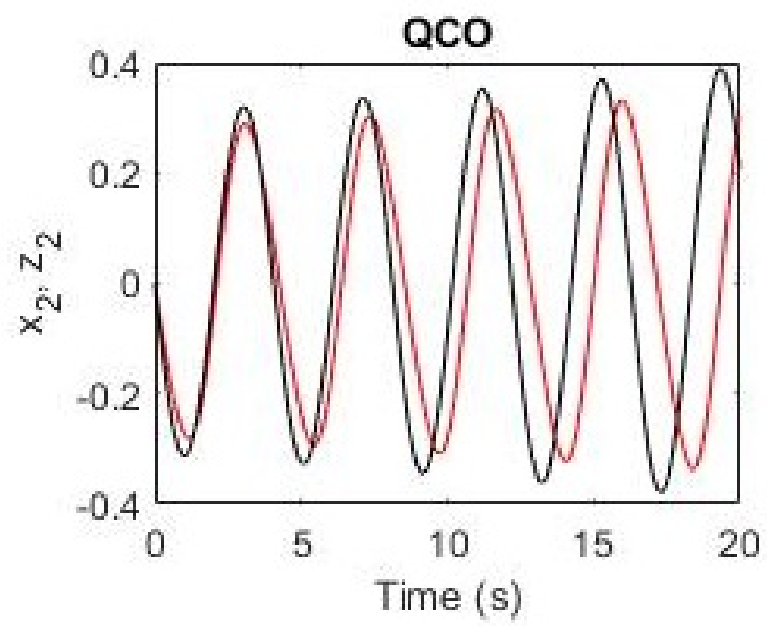

Fig. 8. Parameter $\mathrm{s}$ is reduced: $x_{2}$ (red) and $z_{2}$ from initial condition $x_{2}(0)=0, z_{2}(0)=0$. There is an increase in frequency in relation with case 1 .

C. Case 3: $g_{1}=-0.5, g_{2}=2.5, s=1.0$

The difference $\left|g_{1}-g_{2}\right|$ is increased, this causes an increase in frequency $\omega$.

Figure 9 shows state variables $\left\{x_{1}, z_{1}\right\}$ and Figure 10 shows state variables $\left\{x_{2}, z_{2}\right\}$ for systems (5) and (9), same initial conditions as before. Taylor linearization for a perturbation around the equilibrium point is a good approximation for the nonlinear system. A comparison between case 1 , figures 2 and 3, and case 3, figures 9 and 10, shows an increase in frequency as predicted (10) by the analysis of the linearized system.

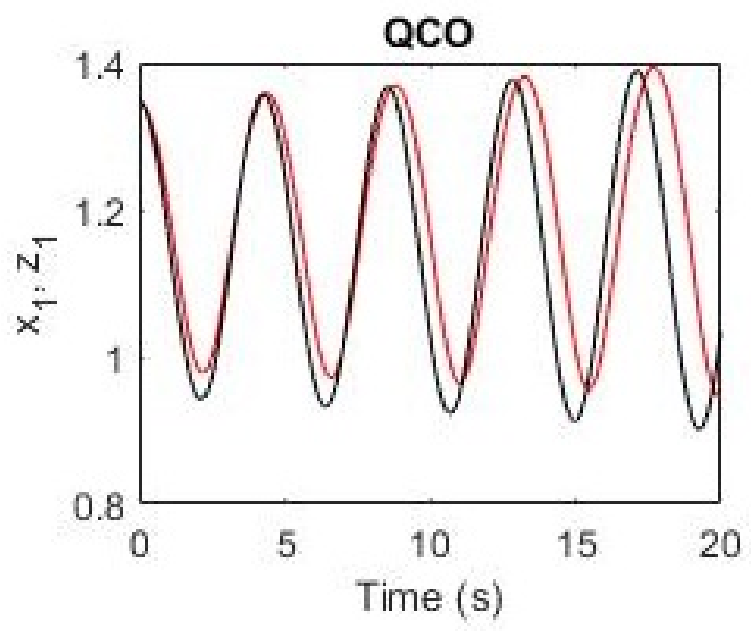

Fig. 9. The difference $\left|g_{1}-g_{2}\right|$ increases: $x_{1}$ (red) and $z_{1}$ from initial condition $x_{1}(0)=\bar{x}_{1}+\Delta x, z_{1}(0)=\Delta x$. There is an increase in frequency in relation with case 1 .

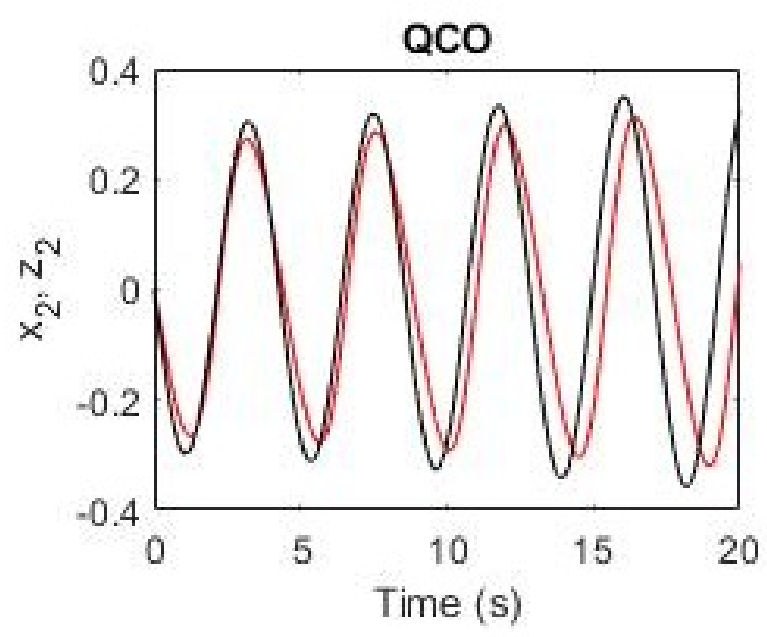

Fig. 10. The difference $\left|g_{1}-g_{2}\right|$ increases: $x_{2}$ (red) and $z_{2}$ from initial condition $x_{2}(0)=0, z_{2}(0)=0$. There is an increase in frequency in relation with case 1 .

\section{CONCLUSIONS}

A parametrized quantum model embedded in a chain of integrators has been used to create an oscillator with tunable frequency, this example illustrates a hybrid dynamic system with a quantum model and a classical dynamic system. The analysis of the nonlinear hybrid system was carried out with Taylor linearization to learn how the parameters $\left\{g_{1}, g_{2}, s\right\}$ can change the oscillation frequency, two conditions were found for $\left\{g_{1}, g_{2}\right\}$ to have an oscillator. 
The dynamic system developed in this paper suggests that it is possible to analyse quantum models using mathematical tools from classical control theory. Proposing a model and then using simulations to study its capabilities creates understanding for the integration of quantum and classical conceptual frameworks.

\section{ACKNOWLEDGMENT}

The author, Alberto Delgado, thanks his employer The National University of Colombia at Bogota for supporting his research.

\section{REFERENCES}

[1] J. Preskill, "Quantum computing 40 years later," arXiv:2106.10522, 2021.

[2] M.A. Nielsen, and I.L. Chuang, Quantum Computation and Quantum Information. Cambridge: Cambridge University Press, 2010.

[3] National Academies of Sciences, Engineering, and Medicine. Quantum Computing: Progress and Prospects. The National Academies Press, Washington, DC, 2019.

[4] J. Preskill, "Quantum Computing in the NISQ era and beyond," arXiv:1801.00862, 2018.

[5] B. Marr, Artificial Intelligence in Practice. Wiley, Cornwall - UK, 2019.

[6] M. Cerezo, A. Arrasmith, R. Babbush, S.C. Benjamin, S. Endo, K Fujii, J.R. McClean, K. Mitarai, X. Yuan, L. Cincio, P.J. Coles, "Variational quantum algorithms," arXiv:2012.09265, 2020.

[7] M. Schuld, "Supervised quantum machine learning models are kernel methods," arXiv:2101.11020, 2021.

[8] A. Delgado, "Function approximation with quantum circuit," arXiv: $1804.00557,2018$.

[9] A. Delgado, "Quantum memristor," TechRxiv.12489149.v2, 2020.

[10] H.K. Khalil, Nonlinear Systems. Macmillan Publishing Company, Singapore, 1992. 